\title{
Developing Unique Engineering Solutions to Improve Patient Safety
}

\author{
Bradley V. Watts, MD, MPH ${ }^{1,2,3}$; Douglas Van Citters, PhD $^{4}$; \\ Brian Shiner, MD, MPH ${ }^{2,3 *}$; Peter D. Mills, PhD, $\mathbf{M S}^{1,3}$ \\ ${ }^{1}$ VA National Center for Patient Safety, White River Junction, VT, USA \\ ${ }^{2}$ Dartmouth Medical School, Hanover, NH, USA \\ ${ }^{3}$ New England Veterans Engineering Resource Center, White River Junction, VT, USA \\ ${ }^{4}$ Thayer School of Engineering at Dartmouth College, Hanover, NH, USA
}

Submitted January 2012. Accepted for publication April 2012.

\begin{abstract}
Many efforts to improve healthcare safety have focused on redesigning processes of care or retraining clinicians. Far less attention has been focused on the use of new technologies to improve safety. We present the results of a unique collaboration between the VA National Center for Patient Safety (NCPS) and the Thayer School of Engineering at Dartmouth College. Each year, the NCPS identifies safety problems across the VA that could be addressed with newlyengineered devices. Teams of Thayer students and faculty participating in a senior design course evaluate and engineer a solution for one of the problems. Exemplar projects have targeted surgical sponge retention, nosocomial infections, surgical site localization, and remote monitoring of hospitalized patients undergoing diagnostic testing and procedures. The program has served as an avenue for engineering students and health care workers to solve problems together. The success of this academic-clinical partnership could be replicated in other settings.
\end{abstract}

Keywords: healthcare, patient safety, education, engineering design process, public-private sector partnerships

\section{INTRODUCTION}

Beginning with the publication of the "To Err is Human: Building a Safer Health Care System" [1], patient safety has been recognized as major problem in the US health care system. That report summarized nearly twenty years of research demonstrating that patients are frequently harmed when receiving healthcare, and has served as a call to

*Corresponding author: Brian Shiner, White River Junction VA Medical Center, 11Q, 215 North Main Street, White River Junction, VT 05009. Phone: (802) 295-9363 x6036. Fax: (802) 291-6286. Email: brian.shiner@va.gov. Other authors: bradley.watts@va.gov; douglas.vancitters@dartmouth.edu; peter.mills@va.gov. 
action for the health care system. Considerable focus and efforts have been brought to bear in efforts to improve the safety of health care. Unfortunately, the evidence over the decade since "To Err is Human" suggests that any gains in promoting safety have been modest at best [2].

There have been a variety of approaches attempting to improve patient safety. Much of the initial effort focused on developing a better awareness of adverse events and attempting to learn from the failures. Root Cause Analysis represents the best known example of this approach [3]. A deeper understanding of human factors engineering has also been applied to both understand and mitigate patient safety problems [4]. At the same time, understanding medical communication and the functioning of health care workers as a team has proven to be a powerful lens for understanding medical errors and for developing successful interventions to improve patient safety [5]. Underlying all of the efforts to promote safer health care is the desire to build a "culture of safety" among all health care workers [6].

There has been substantial attention to the issue of design in the many efforts to improve patient safety. A conceptual framework has been put forward as a means to both understand error and develop better designs to avoid error [7]. Health care teams also develop ideas for new devices and new designs in their efforts to provide safer care [8]. In addition to the theoretic framework and the practical attempts to improve safety, there is a growing literature which suggests differential effectiveness of various types of solutions for patient safety problems $[9,10]$. Some interventions such as policy changes may be less effective than changes to the devices and environment where care is provided [11].

In 2008, the Veterans Administration's (VA) National Center for Patients Safety (NCPS) entered into a unique partnership with the Thayer School of Engineering at Dartmouth College. As a part of this collaboration, the VA would bring forward safety challenges facing the organization and the Thayer School of Engineering's students and faculty would help engineer possible solutions. The NCPS would remain engaged with the design team through regular meetings, and would provide the engineers with access to clinical areas and expertise. In addition, the NCPS would provide funding for the materials needed for the projects. The Thayer Engineering teams take on one to two new projects per academic year. This report describes the initial five projects undertaken by student teams and their impact on operations in the VA.

Highlighted are the project selection process and the breadth of problems that can be solved in this fashion. Specifically, projects include a chemical engineering approach to addressing retained surgical sponges; a mechanical engineering approach to sterilization of reusable medical equipment; a biotechnology approach to identifying infectious agents on surfaces; an imaging and process approach to address wrong site surgery; and a software approach to monitor patient status during activities of daily living.

\section{METHODS}

\subsection{Project Selection}

The NCPS undergoes a rigorous process each year to attempt to indentify safety problems which could be addressed with newly engineered devices and processes. NCPS staff queries the patient safety managers at each of the VA's 156 medical centers to indentify safety issues that could be approached in this manner. In addition, NCPS 
staff reviews a national database of adverse events in an attempt to identify other patient safety problems amenable to design solutions. Typically NCPS receives approximately fifty safety problems from its query of patient safety managers, and an additional ten problems are gleaned from the adverse event database. Projects are selected based on a prioritization matrix [9].

\subsection{Academic Course Structure and Team Formation}

Project abstracts are submitted to the Cook Engineering Design Center (CEDC) at Dartmouth's Thayer School of Engineering. The CEDC serves as a liaison between the engineering school and industrial partners in order to bring real problems into the educational arena, specifically the capstone senior design course. In general, senior design projects at Dartmouth's Thayer School of Engineering are designed to give 4th and 5th year students a real-world engineering experience with guidance from outside professionals. Course lectures relate to professionalism (ethics, intellectual property, problem solving, etc.), and the students are each expected to work about 20 hours a week on their projects for the duration of the 6-month course. The course director therefore coordinates with all potential project sponsors to ensure that project abstracts are of appropriate scope and degree of difficulty for the incoming senior class population. Patient safety project abstracts are modified by NCPS staff as necessary to best fit within the educational goals of the course.

Students in the design course comprise the entire senior engineering class at Dartmouth. While students might self-identify with a traditional engineering discipline (e.g., mechanical, electrical, etc.), the course is only offered once per year, and only in a non-disciplinary format. After a week-long interview and selection process, students prioritize project choices, and multidisciplinary teams of three to four senior undergraduates are assigned to projects by the course instructor. A typical student team would thus include three advanced engineers who are excited about the general field of the project, but who are likely from different engineering disciplines. The students are given laboratory space, a modest budget, and access to all of the Dartmouth facilities to complete their work. Additionally, the students are required to have at least one faculty advisor who is knowledgeable in the chosen field. As a result, the students are constantly interacting with no fewer than three professionals, including the instructor, the faculty advisor, and the NCPS sponsor. The course design is outlined in figure 1 .

Each of the individual project teams applies a defined engineering problem solving method taught at the Thayer School of Engineering at Dartmouth College [12]. The process begins with clinical staff presenting engineers with a broad-based problem statement. The engineers then use a number of predefined steps structured to find the best solution. These steps are common to each of the case examples described in this paper and are briefly described below.

\subsubsection{Identifying the Problem and Need Statement}

Problems presented to an engineer might be biased, might contain errors of fact, and might imply a solution. The presenting clinician may have been involved in the development of the current approaches, and may have preconceived notions as to the best solution. Thus an important initial step is to reconfigure the problem into a true 


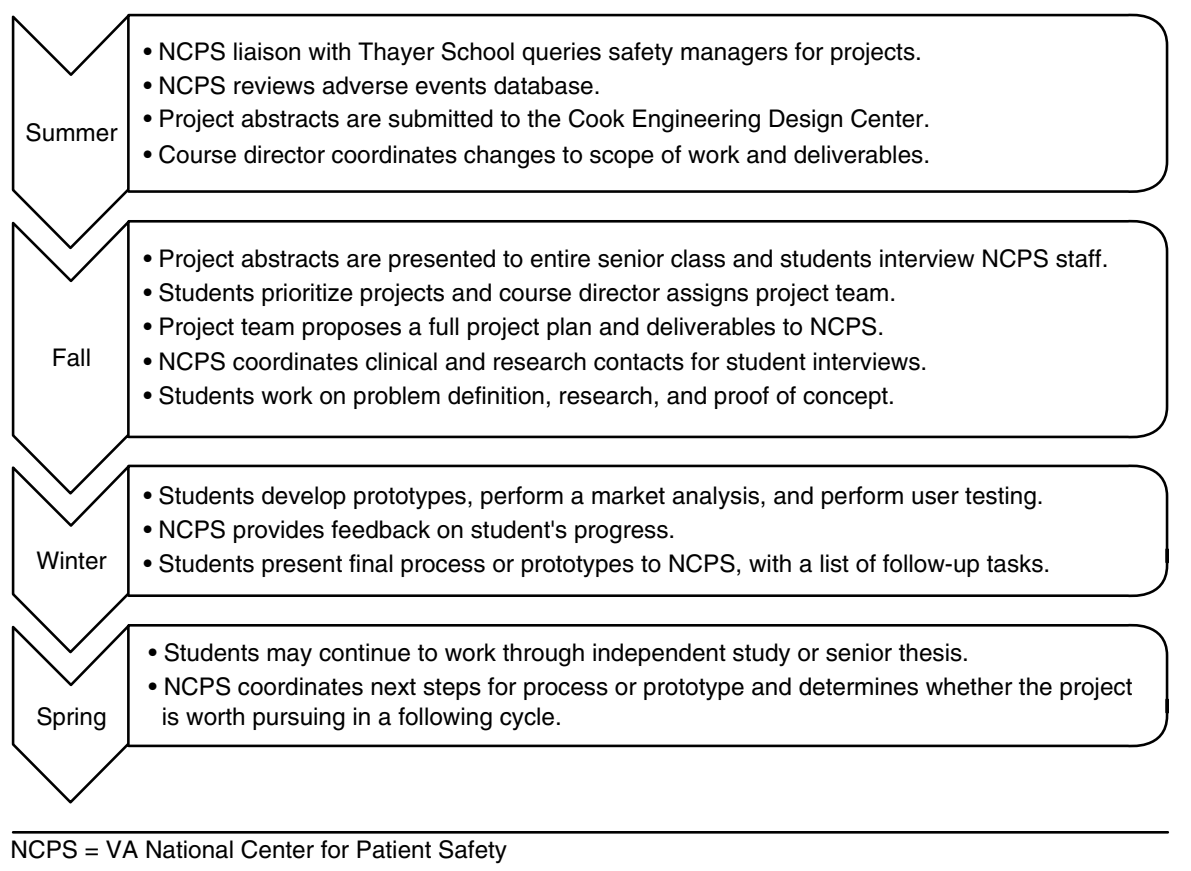

Figure 1. Project course design as it pertains to patient safety-related projects in the VA.

need statement that is without bias and affords maximal freedom for innovation. A reformulated problem statement is used by the engineers to identify the precise deliverables for the project.

The process of developing a need statement and problem statement takes days or weeks, and involves significant amounts of on-site observation, interviews with all stakeholders, and laboratory-based experiments. The engineers are also asked to better quantify the problem as part of their initial research, such that the problem can be placed in the context of the greater societal need.

\subsubsection{Identifying Constraints and Forming into Specifications:}

Understanding the system and the context also requires a thorough understanding of state-of-the-art solutions, their benefits, and drawbacks. All potential solutions are limited or constrained by the limits of current science. In the process of identifying the constraints, the engineering team should identify and evaluate ideas and technologies which have already emerged as potential solutions. These existing solutions are termed the current state-of-the-art. At this step, the engineering team begins to formulate the metrics and methods which will be applied to all new solutions. These metrics should be relevant, measurable, and weighted based on perceived relative importance.

Metrics and specifications must be collected from all stakeholders. Success of a process or product often depends on the ability of the engineer to translate the voice of 
the user, consumer, and patient into the voice of the engineer. Identifying the tradeoffs associated with different specifications permits the engineer to understand the strengths and weaknesses of existing and potential solutions.

\subsubsection{Brainstorming and Analyzing Alternatives}

Brainstorming should be unbiased (with each suggestion openly viewed as a positional solution), and thorough (so that all potential solutions are compared using the predefined specifications). Alternative solutions are evaluated using a logical process which can range in complexity from a "pros-cons" tradeoff chart, to a modified Pugh Decision Matrix, to a House of Quality evaluation [12]. This alternative matrix quantitatively compares each solution using the predefined specifications and their tradeoffs. Each potential solution from the brainstorming session is thus numerically ranked against all others, and the solution with the highest numerical value is chosen as the primary solution path.

\subsubsection{Development of and Testing Chosen Alternative}

The engineering teams dedicate a significant amount of time and work to developing the chosen solution. This step often involves fabrication of a prototype, modeling a solution, or developing a policy. This solution is then compared to the state-of-the-art through laboratory and user testing.

\subsubsection{Analyze Alternative Relative to Problem Statement}

If the chosen solution successfully addresses the specifications and need statement, then the team has solved the problem and reports the findings to the sponsor. Typically, multiple prototypes of the solution are needed before the specifications are adequately met.

\section{RESULTS}

The projects presented below were classified by the White River Junction VA Research and Development Committee as laboratory research not requiring human subjects review. Students obtained pre-approval for formal provider surveys in the retained surgical sponge project from the Dartmouth Committee for the Protection of Human Subjects (CPHS \#22776).

\subsection{Project Areas}

\subsubsection{Retained Surgical Sponge}

Unintentionally leaving surgical items in patients has emerged as a significant patient safety problem facing the health care system. There are reports of different items including scalpels, forceps, and surgical needles being retained in patients' body cavities after surgery [13]. The retention of surgical sponges is among the highestimpact patient safety problems [14]. While ever more elaborate methods have been employed to decrease the risk of leaving objects in patients (such as more complex protocols to count instruments in the surgical field $[15,16])$, there remain a significant number of occurrences [17]. 
The engineering approach focused on avoidance of retention by means other than counting or tracking objects. Initial efforts focused on developing methods to conduct surgery without using sponges. Although this method holds promise (and to some degree was already occurring with the increased use of minimally invasive procedures), it was beyond the scope of this project as it would require retraining large number of surgeons. A second option was developing a sponge which could be safely left in a patient. This became the focus of the project.

The team of students assigned to the project included a biomedical engineer, a chemical engineer, and an economics/engineering double major. Their engineering team developed a sponge composed of combined alginate and cellulose fibers, which harmlessly biodegrade in the human body. This material initially retains the functionality of a traditional cotton sponge, but is absorbed into the body if left in after a procedure. As a result of collaborations with practitioners, the design team also worked to make the material retain the "feel" of a cotton sponge and other aspects valued by surgeons.

The design was awarded second place recognition at the Collegiate Investigators Competition [18], and the investigators have applied for patent protection. The students are the sole named inventors on the application, and as with all student projects performed at Dartmouth, assignment of the patent rights is handled on a case by case basis via the central Technology Transfer Office. Additional work has been dedicated to the manufacturing process and animal studies should begin soon.

\subsubsection{Pathogens in hospitals}

The transmission of infectious organisms within the hospital setting is likely the most significant patient safety problem involving inpatient medical care. A great deal of work has been done already to design clinical protocols to avoid the development of nosocomial infections [19]. These have ranged from hand washing campaigns to the use of checklists to ensure compliance with procedures known to decrease the incidence of catheter-related infections, surgical site infections, and ventilator-associated pneumonia [20, 21].

Two teams took different approaches to impact various aspects of this problem. The first team addressed the scenario in which there is a transmission of an infectious agent from a known infected patient maintained in isolation. Hospital staff wear disposable gowns and gloves when in these areas. However, reusable medical equipment such as stethoscopes, reflex hammers, and glucometers may not be adequately cleaned after being exposed to this environment. The current practice involves the time-intensive process of wiping the device or instrument with alcohol-based cleaners.

The student team assigned to this project included a mechanical engineer, a bio-engineer, and a design engineer. The team initially focused on observing the care process, specifically, the types of devices used and the process by which the health care workers left the room and took off their gloves and gowns. Based on these observations, they developed specifications for a device that could be located in the hall outside a patient room and could sterilize objects in less than 30 seconds. The team constructed a device that was small enough to fit on the cart containing disposable gowns and 
gloves outside the patient's room. It could be opened using a foot pedal and disinfects medical equipment using ultra violet light in 30 seconds. The ultraviolet dose rate of the device exceeds $4,800 \mu \mathrm{W} \cdot \mathrm{s} / \mathrm{cm}^{2}$ per second, allowing for disinfection sterility assurance levels better than $10^{-3}$. Future work will focus on cost effectiveness and a scalable manufacturing process.

A parallel area of exploration was the scenario in which there is a need to detect infectious agents on surfaces. A student team including two bio-engineers, a chemical engineer, and a biomedical engineer set out to develop a rapid method to accurately identify specific pathogens. Such a device would allow hospital personnel to rapidly determine whether a surface is disinfected. Their goal was to develop a portable handheld device that could, in less than 30 seconds, detect bacteria on surfaces. In addition, clinician feedback indicated that it would be desirable if the device could identify specific pathogens of interest, such as methacillin-resistant Staphylococcus aureus or Clostridium difficile.

The project employs molecular beacons and a fluorescence detector in conjunction with a microfluidics platform to achieve better than $95 \%$ sensitivity, and less than $5 \%$ false positives. Sample swabs from surfaces in question are introduced into the testing device. Microporation is used to introduce the molecular beacon into any pathogens that might be present in the sample. Up to seven samples can be tested simultaneously in a device that is $25 \mathrm{~cm} \times 15 \mathrm{~cm} \times 10 \mathrm{~cm}$, and results can be obtained in less than 10 minutes. While the pilot device was able to accomplish the stated goal, considerable work is needed to refine such issues as power supply and reusability. Consequently, much more development is needed for this project before it is ready for production.

\subsubsection{Wrong Site Spinal Surgery}

Conducting medical procedures on the wrong body part or on the wrong patient is a safety issue that easily captures the attention of the public. While these events are thought to be rare, they do pose significant harm to those patients affected. In recent years, the Joint Commission and most hospitals have developed guidelines for correct identification of surgical sites [22, 23]. These guidelines mostly entail having the surgeon sign the site prior to surgery. While this approach has generally been effective, it has been difficult to apply to some surgical procedures, especially those in which site identification by signing the skin is infeasible. Spinal surgery presents such a situation. In this case, marking the surgical site does not necessarily localize the spinal level or correct side. The current process requires use of an immovable marker whose location is radiologically confirmed. Despite this mandated process, wrong site spinal surgeries continue to occur $[24,25]$.

The student engineering team formed for this project included a mechanical design engineer, a biomedical engineer, and bio-engineer. They sought to establish a method by which the correct surgical site, including spinal level and laterality could be determined before the surgery in a way that would be obvious to the surgical team during the procedure. In this case, rather than designing a new product or device, the team simply applied an existing technology to a new application. Localization wires are commonly used in breast surgery to localize a lesion through computerized tomography 
(CT) guidance and provide a visual guide to the surgical team. The student team conducted initial feasibility studies to explore the possible application of this technology to spinal surgery. They developed a process through which a herniated disk would be localized and marked using a localization wire by a radiologist using CT guidance. The first step in this process was to localize the lesion using CT imaging. The radiologist would then introduce the guide wire under CT guidance. The guide wire would terminate at the location of the intervertebral disk pathology and extend through the skin. The exposed guide wire would be covered and bandaged. Hours later, a surgeon would use the guide wire to localize the correct disk for surgical intervention. The student team's development work employed cadaveric pigs in the radiology suite at Dartmouth-Hitchcock Medical Center as well as feedback from surgeons on the utility of the approach.

The team executed the process described above with actual radiology and surgical staff using a cadaveric pig. The disk could be localized and marked using the wire, and the surgeon could easily follow the wire to the correct disk space. As all the basic equipment is available and FDA approved, the team is moving forward with plans to do a pilot study using this process in actual patients undergoing spinal surgery. Two potential problems with this approach were identified by the team: movement of the location wire and potential of infectious agents to enter the body through the location wire track. Preliminary testing suggested that movement of the wire is unlikely if correctly implanted. However, evaluation of the risk of infection remains an area needing additional work.

\subsubsection{Continuous Monitoring of Hospitalized Patients}

Hospitals are large complex health care delivery sites. Patients receiving care in hospitals may, during a particular day, receive care in multiple locations within a hospital such as radiology, operating rooms, and physical therapy clinics. In addition, patients may spend considerable time in transition or waiting in these locations. Hospitals lack reliable means to know the location of all patients at all times. Furthermore, patients could decompensate clinically while in an ancillary testing area, where their worsening status could go unnoticed.

The goal of this project was to develop a means of knowing the location and basic physical status of all patients at all times [16]. Because hospitals are typically multileveled structures, the location must include a three-dimensional specification. In addition to knowing where patients are located, an optimal system must also include some monitoring of their health status. The student team assigned to this project included a systems engineer, a computer engineer, and an electrical engineer. The system developed by the student team used wireless internet networks to correctly locate patients. The small device worn by all patients would also capture basic health status data such as heart rate, respiration, and temperature. This supports the rapid identification of signals that indicate worsening health status, which are communicated to clinicians using real-time data displays. Software built into the system also allows for the collection of other health status data. For example, by measuring vertical movement over time (downward velocity), possible patient falls can be detected. 
Much of the current work is focused on developing a user interface that health care workers find appealing and helpful. Obviously, a major consideration is elimination of false positive signals that a patient's health status has worsened. Considerable additional work is needed to ensure that false positive signals do not prove to be a distraction to staff.

\section{DISCUSSION}

Engineering has a long history of significant impact on health care delivery, ranging from the development of advanced prostheses to improving the safety and reliability of surgical anesthesia devices [26-29]. Over the past decade, a great deal of research and attention has been focused on issues of avoiding harm to patients and designing a safer health care system. This has led to increasing numbers of engineering students and faculty from a variety of engineering disciplines who are interested in patient safety. The real innovation described in the cases above is not the development of any of the specific products or processes designed to improve patient safety, but rather the unique ongoing partnership and integration between engineering students and heath care workers to solve new problems. On the surface, such a partnership seems obvious. However, one only needs to consider the myriad problems in health care which have not been investigated and evaluated by engineers to realize the rarity of this approach. That the NCPS is able to identify important areas of focus and quickly enjoy the attention of engineering teams is a unique and valuable aspect of our program. We provide concrete examples of how engineers and health care providers can partner to develop devices and processes leading to safer health care.

The engineering problem solving methodology, as applied to clinical problems, does have some limitations. First, medical products and/or processes require substantial time to move from engineering research and development to clinical practice. For example, the resorbable surgical sponge would require extensive preclinical testing to ensure that it would not lead to problems such as gastrointestinal obstruction before it is resorbed. A solution may take years before implementation. This delay is the primary reason that so many clinical problems are subjected to more rapid trial-and-error problem solving techniques, where the clinician and hospital can quickly observe the outcome of a proposed solution. We have observed that the projects developed through our program provide fast and valuable insight into whether a prototype idea or product is worth the additional time and expense for full development to clinical practice.

The engineering problem solving methodology may also be limited by a lack of clinical knowledge on the part of the consulting engineers. While some healthcare engineering programs require engineering students to take clinical courses, engineers from other traditional disciplines might not have access to clinical coursework. Thus, the engineer must form a working relationship with the clinicians to best understand the scope of the problem and evaluate potential solutions. The current project accommodates this limitation through providing multiple academic and clinical advisors to the engineering team. While these resources might not be available to professional consulting engineers, the structure is readily in place in academic institutions, and professionals are eager to share knowledge and ideas with students. 
Finally, engineering consultation adds further upfront expense to the problem. The resources necessary to fully develop a solution through iterative problem solving and production of prototypes may be substantial. Alternatively, making small changes in the clinic over time through trial and error is at first glance inexpensive, but the costs add up when the problems are not solved. Evaluating the cost of problem solving and defining the market for an eventual product are crucial steps to the engineering approach, and this evaluation should justify the need for thorough upfront development of a solution. The projects developed through this partnership have low financial risk due to an academic partnership on the teaching side, as opposed to the research side. Moreover, the breadth of student experience and educational objectives of the course mandate that cost-benefit analysis for the engineering project is performed.

A drawback to partnerships such as the one described is that projects are of finite length and scope. As identified with the five projects reported in this work, the products, while successful, are not necessarily marketable. The development cycle has the potential to be broken if a responsible party is not identified for the "next steps." However, it might be of benefit to the sponsoring organization to get the students sufficiently excited about patient safety so that they might wish to pursue a career in the field, perhaps starting with continuation of their academic work in a public or commercial setting.

\section{CONCLUSION}

The partnership between NCPS and Dartmouth's Thayer School of Engineering has been a mutually beneficial relationship for all parties. To date, five difficult, multidisciplinary, safety-related problems have been addressed by broadly trained engineering students in a design-focused environment. Success of the program is attributed to an academic environment focused on open collaboration between the engineering school and the clinical partner. The development of partnerships between engineers and health care providers to improve patient safety is a scalable enterprise. Other health care organizations and schools of engineering could likely replicate these promising results.

\section{ACKNOWLEDGEMENTS}

This work was supported by the Veterans Health Administration's National Center for Patient Safety. The views presented here are those of the authors and not necessarily the views of the Departments of Veterans Affairs or of the United States government.

The authors wish to acknowledge the administrative and financial support of the Cook Engineering Design Center (Director: Ronald Lasky, PhD) for facilitating the project selection and resource allocation process. We would like to thank participating students for valued efforts: (1) Retained Surgical Sponge: Nathan Niparko, Devon Anderson, Jonathan Guerrette (Advisor Mark Laser, PhD); (2a) Pathogens in Hospitals (Sterilization): Jasmine Le, Mncedisi Sikhondze, Christabell Makokha (Advisor Karl Griswold, PhD); (2b) Pathogens in Hospitals (Detection): Brittany A. Thomas, Ian Blumenthal, Robin Meyers, Tia Min (Advisor Karl Griswold, PhD); (3) Wrong Site Spinal Surgery: Patrick M. Kennedy, Sara M. Lee, Saryah Azmat (Advisor P. Jack Hoopes, DVM, PhD); (4) Non-Continuous Monitoring of Hospitalized Patients: Christopher Roe, Peter De Boursac, Alexander Magleby (Advisor Kofi Odame, PhD). 


\section{CONFLICT OF INTEREST}

The authors have no conflicts of interest to report.

\section{REFERENCES}

[1] Institute of Medicine. To Err is Human: Building a Safer Healthcare System. National Academies Press, Washington, DC, 2000.

[2] Landrigan CP, Parry GJ, Bones CB, Hackbarth AD, Goldmann DA, Sharek PJ. Temporal trends in rates of patient harm resulting from medical care. New England Journal of Medicine. 2010, 363(22): 2124-2134.

[3] Bagian JP, Lee C, Gosbee J, DeRosier J, Stalhandske E, Eldridge N, Williams R, Burkhardt M. Developing and deploying a patient safety program in a large health care delivery system: you can't fix what you don't know about. Joint Commission Journal on Quality Improvement. 2001, 27(10): 522-532.

[4] Bagian JP. Health care and patient safety: The failure of traditional approaches - how human factors and ergonomics can and MUST help. Human Factors and Ergonomics in Manufacturing and Service Industries. 2012, 22(1): 1-6.

[5] Neily J, Mills PD, Young-Xu Y, Carney BT, West P, Berger DH, Mazzia LM, Paull DE, Bagian JP. Association between implementation of a medical team training program and surgical mortality. Journal of the American Medical Association. 2010, 304(15): 1693-1700.

[6] Watts BV, Percarpio K, West P, Mills PD. Use of the Safety Attitudes Questionnaire as a measure in patient safety improvement. Journal of Patient Safety. 2010, 6(4): 206-209.

[7] Norman DA. The Design of Everyday Things. Basic Books, New York, 1988.

[8] Wu AW, Lipshutz AK, Pronovost PJ. Effectiveness and efficiency of root cause analysis in medicine. Journal of the American Medical Association. 2008, 299(6): 685-687.

[9] Bagian JP, Gosbee J, Lee CZ, Williams L, McKnight SD, Mannos DM. The Veterans Affairs root cause analysis system in action. Joint Commission Journal on Quality Improvement. 2002, 28(10): 531-545.

[10] DeRosier J, Stalhandske E, Bagian JP, Nudell T. Using health care Failure Mode and Effect Analysis: the VA National Center for Patient Safety's prospective risk analysis system. Joint Commission Journal on Quality Improvement. 2002, 28(5): 248-267, 209.

[11] Watts BV, Young-Xu Y, Mills PD, DeRosier J, Kemp J, Shiner B, Duncan WE. An Examination of the Effectiveness of a Mental Health Environment of Care Checklist in Reducing Suicide on Inpatient Mental Health Units. Archives of General Psychiatry. 2012, Jun 1, 69(6): 588-929.

[12] Frye E. Engineering problem solving for mathematics, science, and technology education. Trustees of Dartmouth College, Hanover, NH, 1996.

[13] Regenbogen SE, Greenberg CC, Resch SC, Kollengode A, Cima RR, Zinner MJ, Gawande AA. Prevention of retained surgical sponges: a decision-analytic model predicting relative costeffectiveness. Surgery. 2009, 145(5): 527-535.

[14] Gibbs VC, Auerbauch AD. The Retained Surgical Sponge. In Making Healthcare Safer: A Critical Analysis of Patient Safety Practices. Edited by Shojania KG, Duncan BW, McDonald KM, Wachter RM. Rockville, MD, Agency for Healthcare Quality and Research, 2001.

[15] Cima RR, Kollengode A, Clark J, Pool S, Weisbrod C, Amstutz GJ, Deschamps C. Using a datamatrix-coded sponge counting system across a surgical practice: impact after 18 months. Joint Commission Journal on Quality and Patient Safety. 2011, 37(2): 51-58.

[16] Ting SL, Tsang AHC. Development of an RFID-based Surgery Management System: Lesson Learnt from a Hong Kong Public Hospital. Journal of Healthcare Engineering. 2012, 3(3).

[17] Greenberg CC, Gawande AA: Retained foreign bodies. Advances in Surgery. 2008, 42: 183-191.

[18] Collegiate Inventors Competition [http://www.invent.org/collegiate/overview.html]

[19] Arias KM. Outbreak Investigation, Prevention, and Control in Health Care Settings: Critical Issues in Patient Safety. Jones and Bartlett Publishers, Sudbury, MA, 2010. 
[20] Eldridge NE, Woods SS, Bonello RS, Clutter K, Ellingson L, Harris MA, Livingston BK, Bagian JP, Danko LH, Dunn EJ. Using the six sigma process to implement the Centers for Disease Control and Prevention Guideline for Hand Hygiene in 4 intensive care units. Journal of General Internal Medicine. 2006, 21 Suppl 2: S35-42.

[21] Lipitz-Snyderman A, Needham DM, Colantuoni E, Goeschel CA, Marsteller JA, Thompson DA, Berenholtz SM, Lubomski LH, Watson S, Pronovost PJ. The ability of intensive care units to maintain zero central line-associated bloodstream infections. Archives of Internal Medicine. 2011, 171(9): 856-858.

[22] Neily J, Mills PD, Eldridge N, Carney BT, Pfeffer D, Turner JR, Young-Xu Y, Gunnar W, Bagian JP. Incorrect surgical procedures within and outside of the operating room: a follow-up report. Archives of Surgery. 2011, 146(11): 1235-1239.

[23] Neily J, Mills PD, Eldridge N, Dunn EJ, Samples C, Turner JR, Revere A, DePalma RG, Bagian JP. Incorrect surgical procedures within and outside of the operating room. Archives of Surgery. 2009, 144(11): 1028-1034.

[24] Devine J, Chutkan N, Norvell DC, Dettori JR. Avoiding wrong site surgery: a systematic review. Spine (Philadelphia PA 1976). 2010, 35(9 Suppl): S28-36.

[25] Mody MG, Nourbakhsh A, Stahl DL, Gibbs M, Alfawareh M, Garges KJ. The prevalence of wrong level surgery among spine surgeons. Spine (Philadelphia PA 1976). 2008, 33(2): 194-198.

[26] Arfanis K, Fioratou E, Smith A. Safety culture in anesthesiology: Basic concepts and practical application. Best Practice and Research Clinical Anesthesiology. 2011, 25: 229-38.

[27] Highsmith MJ, Kahle JT, Bongiorni DR, Sutton BS, Groer S, Kaufman KR. Safety, energy efficiency, and cost efficacy of the C-Leg for transfemoral amputees: A review of the literature. Prosthetics and Orthotics International. 2010, 34(4): 362-377.

[28] Mohammadi H, Mequanint K. Prosthetic aortic heart valves: modeling and design. Medical Engineering \& Physics. 2011, 33(2): 131-147.

[29] Wennerberg A, Albrektsson T. Current challenges in successful rehabilitation with oral implants. Journal of Oral Rehabilitation. 2011, 38(4): 286-294. 


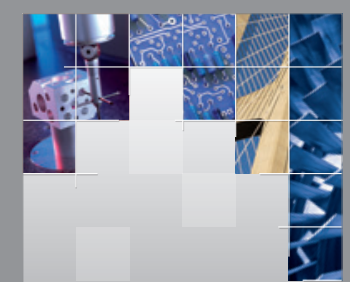

\section{Enfincering}
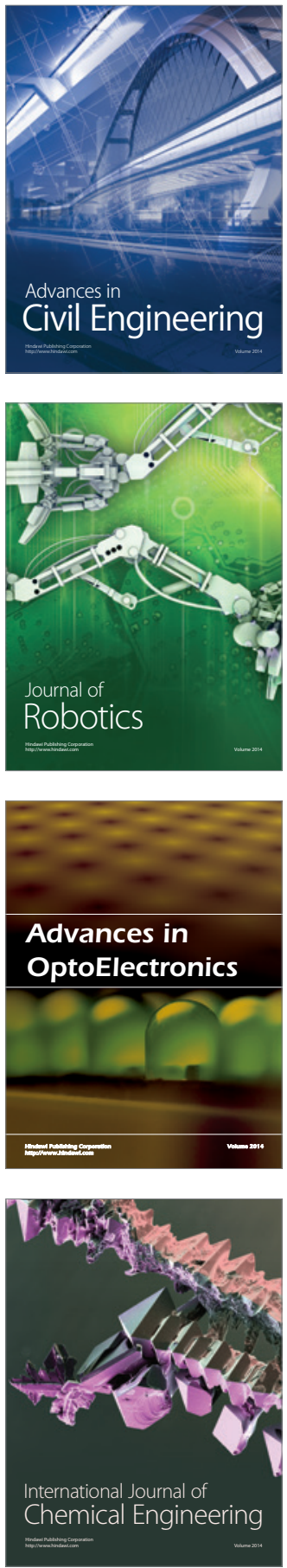

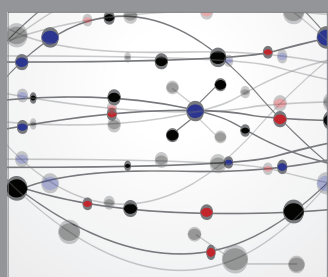

The Scientific World Journal

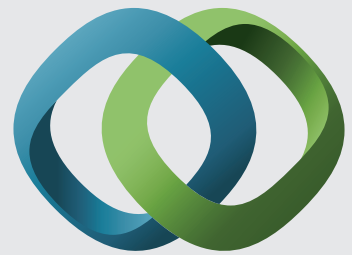

\section{Hindawi}

Submit your manuscripts at

http://www.hindawi.com
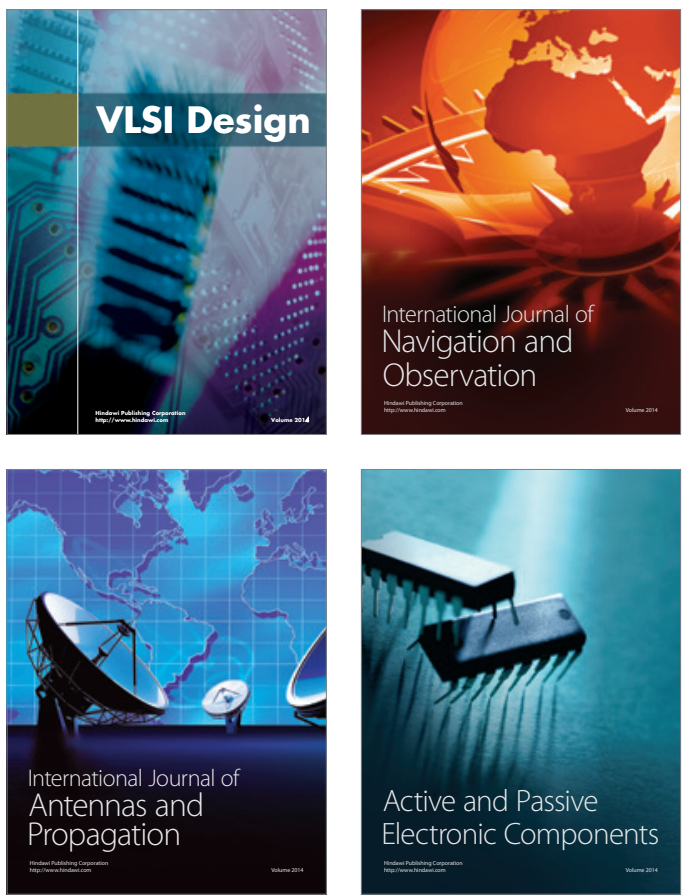
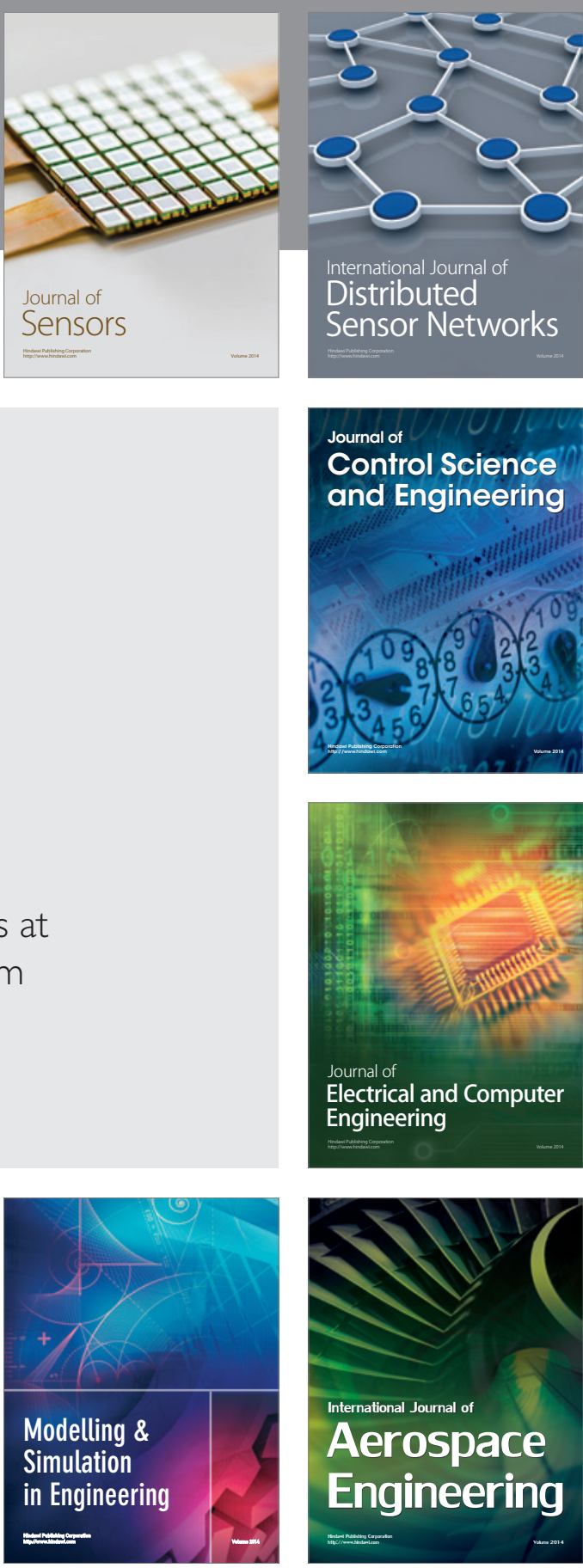

International Journal of

Distributed

Sensor Networks

Journal of

Control Science

and Engineering
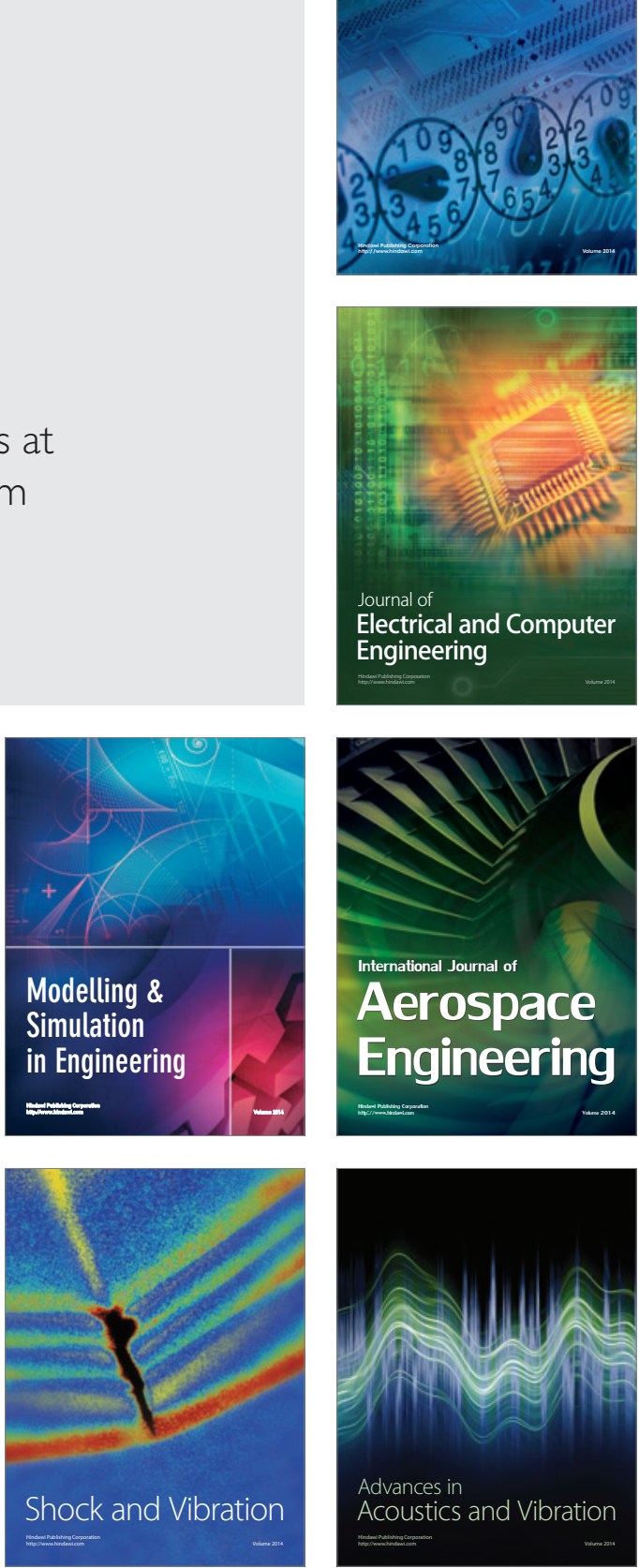